\title{
Dendritic Backpropagation and the State of the Awake Neocortex
}

\author{
Yulia Bereshpolova, ${ }^{1}$ Yael Amitai, ${ }^{1,2}$ Alexander G. Gusev, ${ }^{1}$ Carl R. Stoelzel, ${ }^{1}$ and Harvey A. Swadlow ${ }^{1}$ \\ ${ }^{1}$ Department of Psychology, University of Connecticut, Storrs, Connecticut 06269, and ${ }^{2}$ Department of Physiology, Ben-Gurion University, Beer-Sheva \\ 84105 , Israel
}

The spread of somatic spikes into dendritic trees has become central to models of dendritic integrative properties and synaptic plasticity. However, backpropagating action potentials (BPAPs) have been studied mainly in slices, in which they are highly sensitive to multiple factors such as firing frequency and membrane conductance, raising doubts about their effectiveness in the awake behaving brain. Here, we examine the spatiotemporal characteristics of BPAPs in layer 5 pyramidal neurons in the visual cortex of adult, awake rabbits, in which EEG-defined brain states ranged from alert vigilance to drowsy/inattention, and, in some cases, to light sleep. To achieve this, we recorded extracellular spikes from layer 5 pyramidal neurons and field potentials above and below these neurons using a 16-channel linear probe, and applied methods of spike-triggered current source-density analysis to these records (Buzsáki and Kandel, 1998; Swadlow et al., 2002). Precise retinotopic alignment of superficial and deep cortical sites was used to optimize alignment of the recording probe with the axis of the apical dendrite. During the above network states, we studied BPAPs generated spontaneously, antidromically (from corticotectal neurons), or via intense synaptic drive caused by natural visual stimulation. Surprisingly, the invasion of BPAPs as far as $800 \mu \mathrm{m}$ from the soma was little affected by the network state and only mildly attenuated by high firing frequencies. These data reveal that the BPAP is a robust and highly reliable property of neocortical apical dendrites. These events, therefore, are well suited to provide crucial signals for the control of synaptic plasticity during information-processing brain states.

Key words: corticotectal neurons; fast-spiking neurons; apical dendrites; CSD; spike-triggered averaging; dendritic integration

\section{Introduction}

Most neurons in the mammalian neocortex are "pyramidal" in morphology, characterized by an apical dendrite that ascends vertically to the cortical surface. Recently, it has become clear that action potentials generated near the cell body propagate not only forward along the axon but also backwards up the apical dendrite, and that these back propagating action potentials (BPAPs) may be crucial to synaptic integration and plasticity (Magee and Johnston, 1997; Markram et al., 1997; Linden, 1999; London and Häusser, 2005; Waters et al., 2005). However, our understanding of BPAPs is derived mainly from in vitro studies that suggest that the degree of backpropagation is highly sensitive to multiple modulating factors: it is curtailed by high firing frequencies (Stuart et al., 1997) or by inhibition (Tsubokawa and Ross, 1996; Larkum et al., 1999b) and boosted by coincident excitation (Stuart and Häusser, 2001). This supposed vulnerability has raised questions about the reliability and role of BPAPs in awake brains, in which intense synaptic activity could suppress them (Rudolph and Destexhe, 2003) or, alternatively, nonlinear mechanisms could reinforce their propagation (Williams and Stuart, 2000).

\footnotetext{
Received May 15, 2007; revised July 3, 2007; accepted July 11, 2007.

This work was supported by National Science Foundation Grant IOB-0445583. We thank Victor Serdyukov for expert hardware and software development.

Correspondence should be addressed to Harvey A. Swadlow, Department of Psychology, University of Connect-

icut, 406 Babbidge Road, Unit 1020, Storrs, CT 06269-1020. E-mail: harvey.swadlow@uconn.edu. D0I:10.1523/JNEUROSCI.2218-07.2007

Copyright $\odot 2007$ Society for Neuroscience $\quad 0270-6474 / 07 / 279392-08 \$ 15.00 / 0$
}

Although it has been shown that somatic spikes can invade the apical dendrites in the intact neocortex (Buzsáki and Kandel, 1998; Larkum and Zhu, 2002; Waters and Helmchen, 2004), little is known about the behavior of BPAPs under different network states. Here, we examine the spatiotemporal characteristics of BPAPs in layer 5 pyramidal neurons in the visual cortex of adult (2-4.5 years), awake rabbits, in which EEG-defined brain states ranged from alert vigilance to drowsy/inattention and, in some cases, to light sleep. During these network states, we studied BPAPs generated spontaneously, antidromically, or via intense synaptic drive caused by natural visual stimulation. Our findings demonstrate that the invasion of BPAPs as far as $800 \mu \mathrm{m}$ from the soma was little affected by network state and only mildly attenuated by high firing frequencies.

\section{Materials and Methods}

Recording and stimulation. Recordings were obtained from monocular and binocular primary visual cortex (V1) of two chronically prepared, adult ( 2 and 4.5 years), Dutch-belted rabbits. Initial surgery was performed under sodium pentobarbital anesthesia using aseptic procedures. After removal of the skin and fascia above the skull, the bones of the dorsal surface of the skull were fused together using stainless steel screws and acrylic cement. A stainless steel rod $(6 \mathrm{~mm}$ in diameter, thinned to 2 $\mathrm{mm}$ in places to conserve space on the skull) was oriented in a rostrocaudal direction and cemented to the acrylic mass. The rabbit was held rigidly by this rod during later surgery and recording sessions. A layer of acrylic cement or silicone rubber always covered the exposed skull between recording sessions, and the silicone rubber was also used to buffer the wound margins from the acrylic cement on the skull. Subsequent 


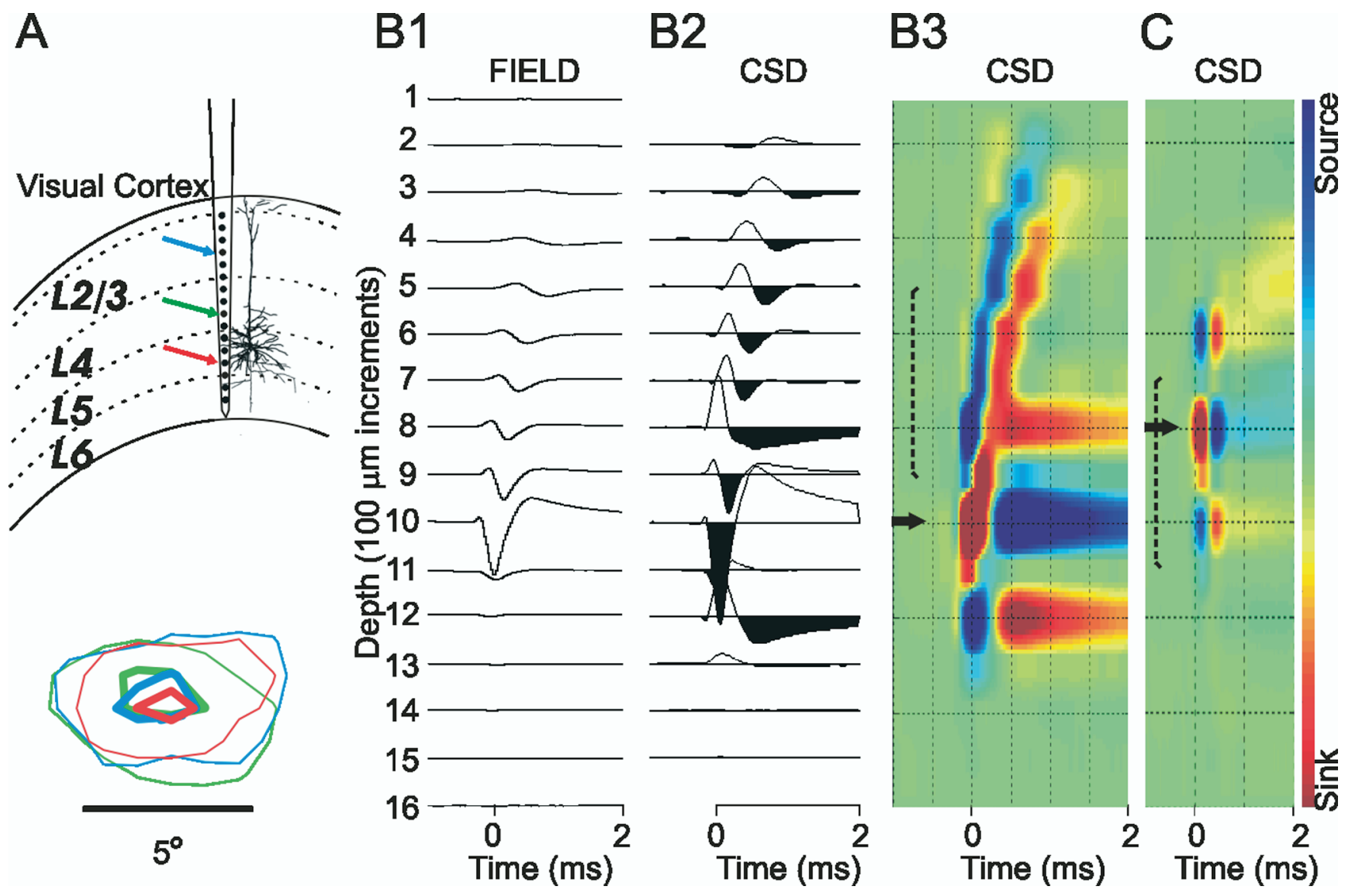

Figure 1. The method for extracellular detection of BPAPs. A, The recording situation. Extracellular somatic spikes were recorded from neurons in layer 5 of V1 of awake rabbits, using one of the recording sites on a 16-channel, vertically oriented linear probe (100 $\mu \mathrm{m}$ spacing; top). Field potentials were recorded from each of the probe sites. Vertical alignment of the probe was guided by simultaneous mapping of visual receptive fields at several recording sites through the cortical layers (blue, green, and red arrows), and the angle of the probe was adjusted until we achieved near-perfect retinotopic alignment (bottom; thick contour lines represent the peak receptive field response, and thin lines indicate contour of the response at $30 \%$ of maximum). B1, Spike-triggered averaging of the field potentials above the somatic recording sites reveals gradually decaying spikes, which are time locked with the somatic spike but with a progressively increasing latency. B2, B3, CSD analysis of the voltage traces delineates a short-duration current sink propagating vertically. The black filled areas in B2 denote current sinks. C, No such backpropagation was seen after the spikes of putative cortical fast-spike interneurons. In all figures, vertical dashed brackets indicate our estimate of the position of layer 4, and the horizontal arrow indicates the recording site of the spike that triggered the CSD profile. Color coding for CSD traces: here, and in subsequent figures, red indicates current sink, and blue indicates source.

recordings were obtained in the awake state through a small entrance in the skull $(<1 \mathrm{~mm})$ using procedures approved by the Institutional Animal Care and Use Committee at the University of Connecticut in accordance with National Institutes of Health guidelines. Methods used to ensure the comfort of our subjects have been described in detail previously (Swadlow et al., 2002). Cortical field potential and multiunit recordings were obtained using 16-channel silicone probes (probe sites separated vertically by $100 \mu \mathrm{m}$; NeuroNexus Technologies, Ann Arbor, MI). These probes are tapered to a fine tip and, at a distance of $2 \mathrm{~mm}$ from the tip, have a width and thickness of 133 and $15 \mu \mathrm{m}$, respectively. Precise alignment of the probe with axis of the apical dendrite was required in these experiments. To achieve this, as a first approximation, simultaneous receptive field maps were obtained from the multiunit activity recorded at superficial and deep probe sites (using a checkerboard pattern of flashing light and dark spots, and methods of real-time, reverse correlation) (Bereshpolova et al., 2006), and the axis of the probe was adjusted, by retracting and reinserting the probe at a slightly different angle, based on the known topography of V1, until the recording sites were in near-perfect alignment. Adjustments in the angle of the probe of as little as $2^{\circ}$ resulted in a noticeable difference in the retinotopic alignment of the superficial and deep recording sites. In some experiments, we controlled the activity level of the recorded neurons using a drifting visual grating and examined BPAPs based on whether the somatic spike occurred during high or low levels of activity. After each recoding session, the entrance in the skull was filled with antibiotic ointment and capped with acrylic cement.
Three or four stimulating electrodes were implanted within the superior colliculus [for antidromic identification of corticotectal (CTect) neurons] (Bereshpolova et al., 2006). These electrodes were constructed of platinum-iridium wire (50-75 $\mu \mathrm{m}$ diameter) and insulated to within $0.5 \mathrm{~mm}$ of the tips. In one of the rabbits, stimulating electrodes were also placed within the retinotopically aligned region of the dorsal lateral geniculate nucleus (LGNd) (for identification of fast-spike, suspected inhibitory interneurons, see Swadlow, 2003). In the second rabbit, a linear array of six stimulating electrodes (oriented along the rostrocaudal axis, separated by $1 \mathrm{~mm})$ was placed within the corpus callosum $(\sim 1.5$ $\mathrm{mm}$ contralateral of the midline) for generating excitatory and inhibitory synaptic drive. The callosal electrodes were constructed of platinumiridium wire (125 $\mu \mathrm{m}$ diameter) and insulated to within $0.5 \mathrm{~mm}$ of the tips. BPAPs of CTect and other layer 5 cortical neurons were monitored by examining the spike-triggered averages of the cortical field potentials, generated from the "spontaneous" activity, visually driven activity, and antidromic spikes.

All stimulating electrodes were implanted under physiological guidance, using initial coordinates guided by the stereotaxic atlas of Sawyer et al. (1954). The final electrode position within the superior colliculus and LGNd was determined by retinotopic alignment with the general area of V1 under study. The position of the callosal stimulating electrodes was adjusted by delivering stimulation pulses as the array was lowered and monitoring low-threshold synaptic responses in the contralateral binocular visual cortex.

Measures of state. We recorded both hippocampal and cortical EEG, 

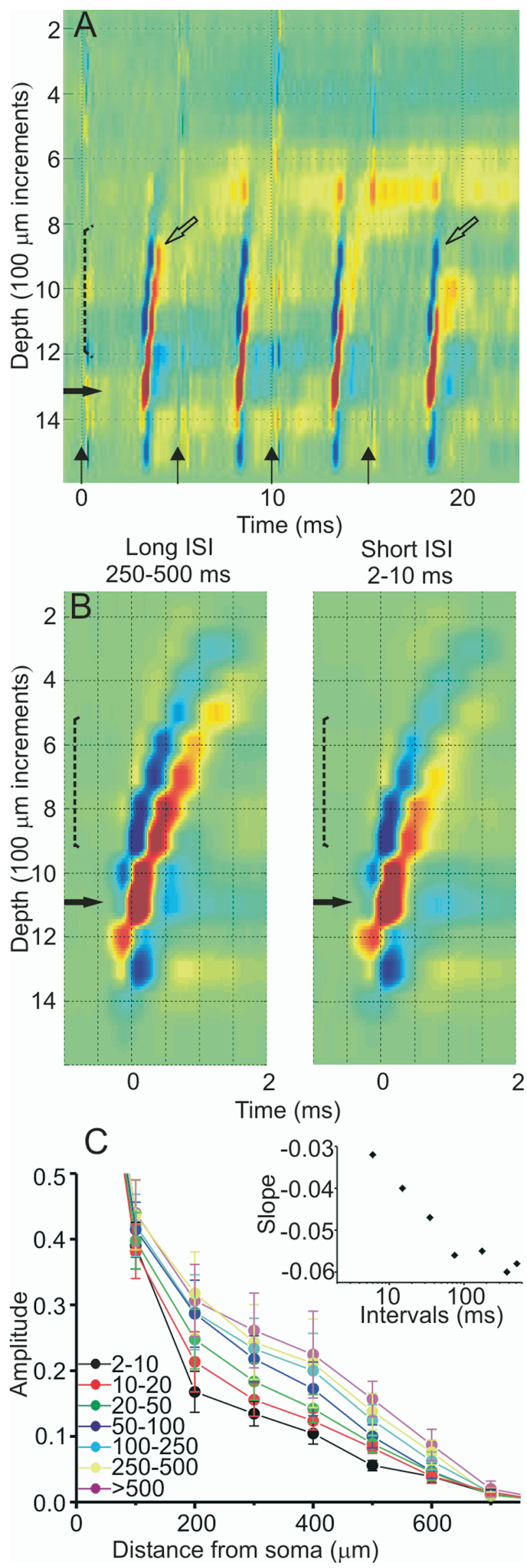

and BPAPs were monitored during four states. (1) The awake, alert state was defined by hippocampal "theta" activity and cortical desynchronized activity. (2) The awake but non-alert state was defined as the $10 \mathrm{~s}$ period immediately after an alert state, during which the hippocampus switches to high-voltage irregular activity, and the cortex switches from desynchronized activity to slow-wave activity (Bezdudnaya et al., 2006). (3) Sleep spindles $(10-16 \mathrm{~Hz})$ are characteristic of light sleep (stages 1 and 2). They are rare in our subjects, but they sometimes occur if subjects are left in a quiet environment for extended periods. (4) The interspindle period refers to the period between two sleep spindles. Hippocampal EEG was obtained via two platinum-iridium microwires placed above and below ( $1 \mathrm{~mm}$ separation) the CA1 layer of the hippocampus. Placement of these electrodes was guided by the characteristic discharge as the pyramidal cell layer is penetrated and by the presence of clear theta activity during arousal. Cortical EEG was recorded using our 16-channel probes (in V1) and using a gold-plated pin placed on the surface of the dura over the somatosensory cortex after identification of this region following receptive field mapping using a finely tapered microelectrode.

Spike-triggered current-source density analyses. Spike-triggered averages of the cortical field activity were generated from the spontaneous firing of layer 5 neurons, many of which were antidromically identified CTect neurons. For the "control" record, we used all of the spikes in the dataset except those that occurred within $5 \mathrm{~ms}$ of another spike. We eliminated spikes with short ( $<5 \mathrm{~ms}$ ) interspike intervals (ISIs) to avoid generating compound averages from any high-frequency spikes. When examining the effects of preceding interspike interval on the spiketriggered potentials, we analyzed only those spikes that were (1) preceded by interspike intervals of a given value and (2) were not followed by another spike within $5 \mathrm{~ms}$. Current-source density (CSD) profiles were generated from the field profiles according to the method described by Freeman and Nicholson (1975). First, we duplicated the uppermost and lowermost field trace (Borroni et al., 1991), which converted our 16 recording channels to a total of 18 channels. Next, we smoothed the traces to reduce high spatial-frequency noise components (Freeman and Nicholson, 1975). This eliminated 2 of the 18 traces:

$$
\text { Smoothing: } \bar{\phi}(r)=\frac{1}{4}(\phi(r+h)+2 \phi(r)+\phi(r=h)) \text {, }
$$

where $\varphi$ is the field potentials, $r$ is the coordinate perpendicular to the layers, and $h$ is the sampling interval $(100 \mu \mathrm{m})$.

Next, we calculated the second derivative, and this yielded a total of 14 traces:

$$
\text { Second derivative: } D=\frac{1}{h^{2}}(\bar{\phi}(r+h)-2 \bar{\phi}(r)+\bar{\phi}(r-h)) \text {. }
$$

In the CSD profiles, current sinks are indicated by downward deflections and sources by upward deflections. To facilitate visualization of CSD profiles, we generated color image plots. These were plotted by linear interpolation along the depth axis, red and blue representing current sinks and sources, respectively. Green is approximately zero, normalized to the $1 \mathrm{~ms}$ period before the cortical spike.

In some experiments, we controlled the activity level of the recorded neurons using a drifting visual grating and generated spike-triggered depth profiles based on whether they occurred during high or low levels of activity (based on where they occurred within the poststimulus time

Figure 2. The effect of firing frequency on the BPAP. A, Reduction in BPAPs during highfrequency $(200 \mathrm{~Hz})$ antidromic activation. Four stimuli are delivered to the superior colliculus (vertical arrows). Open arrows mark the same depth for BPAPs resulting from the first and fourth antidromic spikes in the train for comparison. B, Examples of spike-triggered averaged CSD selected by the interval from the preceding spike (marked above), revealing faster decay for the shorter intervals. C, Summary diagram of the BPAP decays with distance from the soma for different preceding intervals, measured from the spike-triggered averaged CSD and normalized to the somatic spike. Data from 10 neurons are expressed as mean \pm SEM. The inset displays the relationship between the slopes of the linear fits to the decay curves and the mid-bin $|S|$, revealing strong correlation for frequencies higher than $10 \mathrm{~Hz}$. 
histogram) (see Fig. 3). We also analyzed BPAPs that were elicited in CTect neurons by antidromic activation. In these cases, averages of the cortical field activity were generated from the electrical stimulus, which was time locked to the CTect spike. To measure BPAPs evoked antidromically we used only cases in which (1) the antidromic firing threshold was sufficiently low such that the stimulation did not result in elicited field that would obscure the BPAP, or (2) we could stimulate reliably at an intensity that was just below threshold for evoking a spike in our cell. In these later cases, the averaged CSD of the evoked field was subtracted from the suprathreshold CSD to isolate the BPAP. In all cases, at least 50 action potentials were used to generate spike-triggered (or stimulus-triggered) CSD profile.

\section{Results}

\section{Extracellular detection of BPAPs}

We recorded extracellular spontaneous somatic spikes from neurons in layer 5 of V1 of awake rabbits, using one of the recording sites on a 16-channel, vertically oriented linear probe (100 $\mu \mathrm{m}$ spacing) (Fig. 1A, top). Spike-triggered averaging of the field potentials above the somatic recording sites (Fig. $1 B$ ) revealed gradually decaying spikes at distances of up to 800 $\mu \mathrm{m}$ above the soma. These were time locked with the somatic spike but with a progressively increasing latency (Fig. $1 B 1)$. CSD analysis of the voltage traces (Fig. 1 B2,B3) delineated a short-duration $(\sim 0.5 \mathrm{~ms})$ current sink propagating vertically. The ability to monitor these events was severely curtailed by even slight misalignment of the probe with the vertical axis (e.g., if the centers of the superficial and deep cortical recording sites were offset by a distance equivalent to $25 \%$ of the diameter of the smaller of the two receptive fields). To achieve precise alignment, we monitored the visual receptive fields in upper and lower cortical layers and adjusted the angle of our penetration until we achieved near-perfect retinotopic alignment (Fig. $1 A$, bottom). The current work focuses on such well aligned penetrations, in which the vertically propagating sinks could be monitored at distances of at least 400 and up to 800 $\mu \mathrm{m}$ above the soma. The average conduction velocity of the sink measured for the proximal $500 \mu \mathrm{m}$ was $0.78 \pm 0.17 \mathrm{~m} / \mathrm{s}(n=16)$, very close to previous estimates of backpropagation velocity of action potentials in neocortical apical dendrites (Stuart et al., 1997; Buzsáki and Kandel, 1998). However, the propagation velocity was not uniform, and most neurons displayed a clear deceleration and broadening at more distal locations (Fig. 1B2,B3, top). The broadening of BPAPs in the distal dendrite is consistent with in vitro results in layer 5 pyramidal neurons (Stuart et al., 1997) and in vivo, in rat hippocampal pyramidal neurons (Buzsáki et al., 1996). Based on the spatial and temporal features of these vertically propagating electrical events, we conclude that they provide an extracellular measure of the BPAPs along the apical dendrites of neocortical pyramidal cells (Buzsáki and Kandel, 1998). Interestingly, many neurons exhibited a brief earlier sink just below the soma, consistent with action potential initiation in the initial segment/axon (Figs. $1 B 3,2 B$ ).

$\mathrm{CTect}$ neurons were identified by antidromic activation via stimulation of the superior colliculus. These neurons have notably thick apical dendrites (Hallman et al., 1988; Kasper et al., 1994), and, as would be expected, in well aligned penetrations nearly all such neurons (55 of 60) showed BPAPs. Moreover, none of 10 putative fast-spike interneurons identified by action potential duration and very brief ISIs (Swadlow, 2003) showed any evidence of backpropagation (Fig. $1 C$ ). We saw no convincing evidence of a forward propagating signal in any cells. 

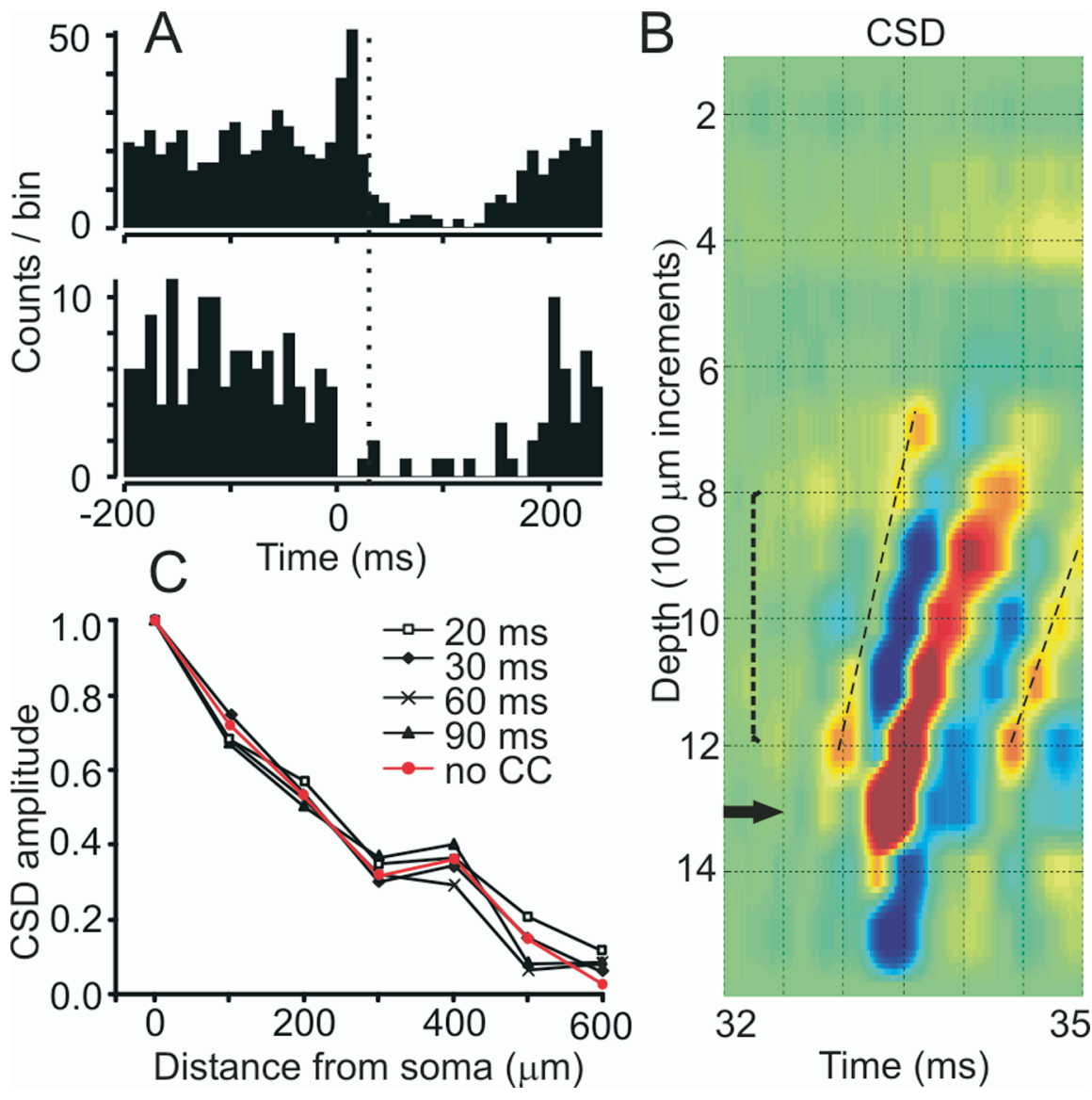

Figure 4. BPAPs evoked during electrically evoked inhibition. $\boldsymbol{A}$, Poststimulus time histograms of the response to callosal stimulation. The top presents multiunit activity from a supragranular electrode site, demonstrating short increase followed by a strong reduction of firing rate. The bottom is from a deeper site, from the CTect neuron studied in $\boldsymbol{B}$, which responded only with prolonged inhibition to callosal stimulation. Inhibition (reduced responding) is seen in the multiunit record throughout the depths of the cortex at intervals of $30-150$ ms after callosal stimulation. The dotted line delineates the timing of the tectal (antidromic) stimulation shown in $\boldsymbol{B}$. $\boldsymbol{B}$, Electrical stimulation of the superior colliculus $30 \mathrm{~ms}$ after the callosal stimulus resulted in an antidromic response 3.3 ms later, during the cortical inhibitory period generated by the callosal stimulus. The resulting BPAP (displayed) is virtually identical to that seen without the callosal stimulus. The oblique dotted lines denote what are probably antidromically elicited BPAPs from other (more distant) CTect neurons. $\boldsymbol{C}$, Plot of the decay curves of the BPAP for the same cell as in $\boldsymbol{B}$, at different time points after the callosal stimulus, and for antidromic spikes evoked with no preceding callosal stimulation (red). CC, Corpus callosum.

\section{The effect of firing frequency on BPAPs}

We next explored which parameters affect the BPAP in the intact awake brain. Studies of both cortical and hippocampal neurons in slices demonstrate significant attenuation of the BPAP amplitude in distal dendrites with increasing firing frequency, manifested at $25 \mathrm{~Hz}$ (Spruston et al., 1995; Stuart et al., 1997), and resulting in frequency-dependent failures at $100 \mathrm{~Hz}$ (Callaway and Ross, 1995). However, another study inducing a more "physiological" firing pattern suggest that a variable firing frequency such as exists in vivo would allow robust BPAPs attributable to an interplay between recruitment and inactivation of the dendritic sodium channels (Williams and Stuart, 2000). We examined this issue in CTect neurons by evoking trains of four antidromic action potentials at varying frequencies. Although the BPAPs were partially obscured by synaptic activity evoked by our stimulation, it was obvious that, even at frequencies as high as $200 \mathrm{~Hz}$, the BPAP was not failing (Fig. $2 A$ ), although the extent of propagation was somewhat reduced. Notably, the BPAPs resulting from low-frequency antidromic activation did not differ significantly from those generated by spontaneous spikes. We next examined the naturally occurring trains of spikes and examined BPAPs of spikes with differing preceding ISIs. For each interval, we plotted the amplitude of the BPAPs normalized to the somatic spike amplitude. We found that the BPAPs generated by high-frequency spikes (short preceding intervals) were only moderately weakened when compared with low-frequency spikes (Fig. $2 B$ ). Figure $2 C$ displays the averaged decay curves ( $n=10$ neurons) and demonstrate a gradual and monotonous strengthening of the BPAP with increasing ISIs. To further examine the effect of the ISI on the BPAP, the decay curves between 200 and $700 \mu \mathrm{m}$ were approximated with a linear fit, and the slope of each fit was plotted against its mid-bin ISI (Fig. 2C, inset). The plot confirms a correlation between the firing frequency and the extent of backpropagation up to firing frequencies of $\sim 10 \mathrm{~Hz}$ and little effect on the backpropagation at slower frequencies.

The effect of synaptic drives on BPAPs In vitro studies indicate that the BPAP is highly sensitive to coincident synaptic events, because excitation boosts and inhibition curtails the degree of the backpropagation (Tsubokawa and Ross, 1996; Larkum et al., 1999a,b; Stuart and Häusser, 2001). We used two types of stimulation paradigms to explore the polarized effects of such synaptic drives in the awake brain. First, we examined the effect of strong sensory excitatory drive by using a drifting visual grating over the receptive field of the pyramidal neuron under study. Decay of the BPAP along the apical dendrites was compared between spikes occurring during the peak of the activation and spikes occurring during the trough $(n=3)$ (Fig. 3). If excitation had a boosting effect, we would expect it to counteract the downgrading effect of the higher frequency. Instead, in each of the three cases examined, BPAPs were mildly reduced during the peak of the activation, suggesting that the firing frequency is the dominant factor (Fig. 3C). Next, we examined the effect of inhibition on antidromically elicited BPAPs. Electrical stimulation of the corpus callosum results in short-latency excitation followed by strong inhibition through the depths of binocular visual cortex that lasts for 150-200 ms (Swadlow, 1974), and this can be verified by a strong reduction of single-unit firing rate at multiple recording sites (Fig. 4A). Antidromic spikes were elicited in CTect neurons at several time points during the inhibitory phase, and all of them resulted in clear backpropagation. Figure $4 B$ shows the antidromically activated BPAPs of a CTect neuron. When compared with antidromic spikes evoked at random, we found, surprisingly, no evidence for weakening of the BPAPs evoked during inhibition (Fig. $4 C$ ). Similar results were seen in each of 11 CT neurons tested in this way. 

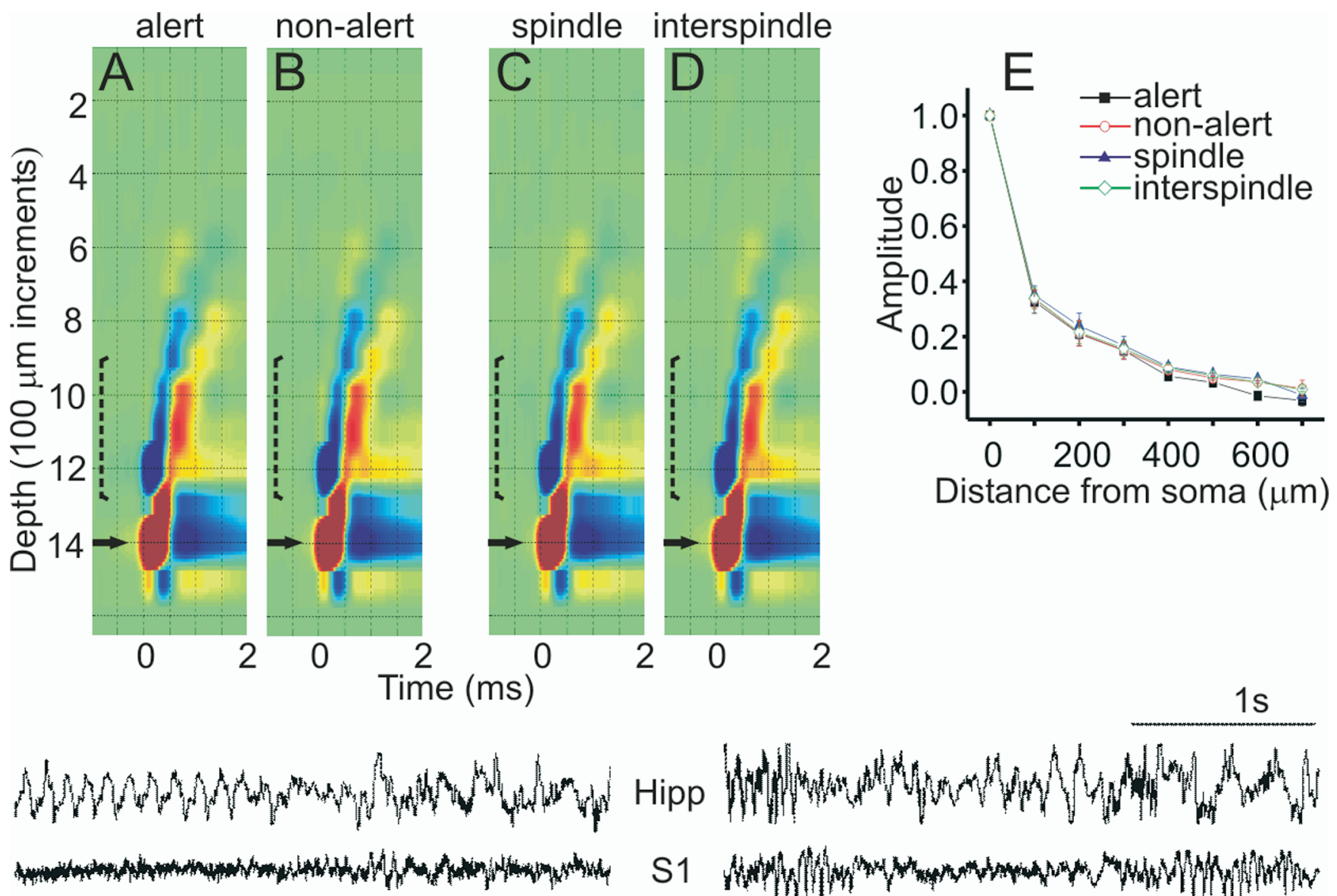

\section{S1}
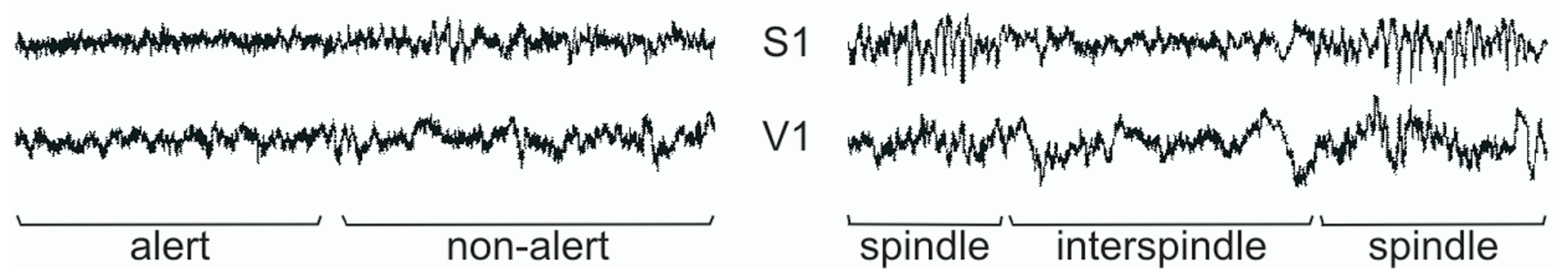

Figure 5. BPAPs during EEG-defined brain states. Specific EEG states (shown below) were identified via recordings from the hippocampus (Hipp), primary somatosensory (S1), and V1 cortex. $\boldsymbol{A}-\boldsymbol{D}$, The BPAPs shown resulted from spikes that occurred during alert vigilance $(\boldsymbol{A})$, an awake, non-alert state $(\boldsymbol{B})$, during cortical sleep spindles $(\boldsymbol{C})$, and during interspindle intervals $(\boldsymbol{D})$. The last two stages are typical of light sleep. $E$, Average decay curves of BPAPs from five neurons during the four states as above. Data are expressed as mean \pm SEM of the CSD amplitude normalized to the somatic value.

\section{The effect of EEG-defined states on BPAPs}

Finally, we compared the BPAPs during several network states as defined by distinct EEG activity (Fig. 5A-D): (1) an alert, attentive state, (2) an awake but non-alert state, (3) during sleep spindles, and (4) during interspindle intervals (the latter two states occur during light sleep). Remarkably, these dramatic changes in network activity had no apparent influence on BPAPs. Figure 5 displays an example of the BPAP during each of these four states, showing compelling similarity. The lack of change in the extent of the backpropagation between the different states is further highlighted in the summary diagram that plots the averaged decay curves of the BPAP across five neurons (Fig. $5 E$ ).

\section{Discussion}

We show here an extracellular record of action potentials propagating from the soma and into the upper reaches of the apical trunk of single cortical neurons in the alert, drowsy, or lightly sleeping brain. Surprisingly, we find that this backpropagation is robust and highly reliable across a wide range of brain states, network activity, and stimulus conditions and is only mildly modulated by neuronal firing frequency.

We are confident that our measures of the BPAP reflect the activity of single dendrites and not the correlated activity of several. Because of the very rapid time course of the BPAP, such correlated activity would need to be extremely precise $(<500 \mu \mathrm{s})$ to influence our measures. However, we observed no such tight synchrony in the spike trains of $>40$ simultaneously recorded pairs of neighboring CTect neurons (Bereshpolova et al., 2006; see also present results). Even "precise synchrony" of thalamic neurons (Alonso et al., 1996) or fast-spike cortical interneurons (Swadlow et al., 1998) occurs over a timescale of $\pm 1 \mathrm{~ms}$, and this would introduce considerable temporal blurring in our measures.

It is important to emphasize that our observations of BPAPs extend only as far as $800 \mu \mathrm{m}$ from the soma, reaching no farther than the upper portion of layer $2 / 3$. Such distances are considerably greater than those observed in a previous extracellular analysis of BPAPs in sensorimotor cortex of awake rats, which used a 
similar cortical recording probe (up to $400 \mu \mathrm{m}$ ) (Buzsáki and Kandel, 1998). The visual cortex of the rabbit (Swadlow and Weyand, 1981) is very similar in thickness to sensorimotor cortex of the rat (both $\sim 1.8-2.0 \mathrm{~mm}$ ) (Paxinos and Watson 1997), so the greater conduction distance of BPAPs seen in this study cannot be attributable to a difference in the length of the apical dendrite. Instead, it is likely attributable to our use of receptive field mapping at multiple cortical depths to maximize alignment of the probe with the axis of the apical dendrite.

It is also important to note that we examined only the relatively short-duration, most likely sodium-dependent, spike and provide no insight into calcium-dependent regenerative events. In vitro studies have demonstrated such events in distal segments of the apical dendrites and mainly at firing frequencies higher than $100 \mathrm{~Hz}$ (Helmchen et al., 1999; Larkum et al., 1999a; Kampa et al., 2006). Technical limitations of our method make the unequivocal detection of calcium spikes difficult: calcium spikes recorded by electrodes display variability in time and amplitude, which would reduce their appearance in a spike-triggered average. Moreover, the slower timescale of calcium events makes it difficult to differentiate them from synaptic sinks resulting from coincident excitatory inputs.

Strong backpropagation in awake brain has been predicted by calcium imaging in vivo (Waters and Helmchen, 2004) and by theoretical work (Rapp et al., 1996) and was demonstrated in awake rats at distances of up to $400 \mu \mathrm{m}$ from the soma (Buzsáki and Kandel, 1998). Interestingly, we find here that not only do the somatic spikes strongly invade the apical dendrites in awake brains, but their degree of propagation under varying conditions is quite uniform. Such reliable backpropagation may be achieved by the electrotonic structure of the trunk and its specific distribution of ion channels (Kim and Connors, 1993; Korngreen and Sakmann, 2000; Lorincz et al., 2002; Waters et al., 2005), such that diverse network dynamics result in stability of the BPAP.

A classical function of dendritic trunks is to convey the results of synaptic processing at distal dendrites to the region of spike initiation near the soma. In addition to this role, our findings support current notions of the apical trunk as a specialized domain for reliably reporting the somatic spike to synaptic sites on distal dendrites. Current theories of spike-timing-dependent synaptic plasticity assume that interactions between synaptic inputs and the BPAP occur during information processing brain states (for review, see Dan and Poo, 2006). Our findings of a remarkable consistency of the BPAPs under a wide range of brain states and sensory/synaptic activation patterns provides essential groundwork for these and other related ideas that implicate BPAPs in synaptic integration and plasticity (Linden, 1999; Waters and Helmchen, 2004; Waters et al., 2005).

\section{References}

Alonso JM, Usrey WM, Reid RC (1996) Precisely correlated firing in cells of the lateral geniculate nucleus. Nature 383:815-819.

Bereshpolova Y, Stoelzel CR, Gusev AG, Bezdudnaya T, Swadlow HA (2006) The impact of a corticotectal impulse on the awake superior colliculus. J Neurosci 26:2250-2259.

Bezdudnaya T, Cano M, Bereshpolova Y, Stoelzel CR, Alonso J-M, Swadlow HA (2006) Thalamic burst mode and inattention in the awake LGNd. Neuron 49:421-432.

Borroni AM, Vaknin G, Berry R, Teyler TJ (1991) Methods for studying the conductance changes associated with synaptic activation of forebrain slices: the interpretation of field potentials using CSD profiles. J Neurosci Methods 39:89-102.

Buzsáki G, Kandel A (1998) Somadendritic backpropagation of action potentials in cortical pyramidal cells of the awake rat. J Neurophysiol 79:1587-1591.
Buzsáki G, Penttonen M, Nádasdy Z, Bragin A (1996) Pattern and inhibition-dependent invasion of pyramidal cell dendrites by fast spikes in the hippocampus in vivo. Proc Natl Acad Sci USA 93:9921-9925.

Callaway JC, Ross WN (1995) Frequency-dependent propagation of sodium action potentials in dendrites of hippocampal CA1 pyramidal neurons. J Neurophysiol 74:1395-1403.

Dan Y, Poo M-M (2006) Spike timing-dependent plasticity: from synapse to perception. Pysiol Rev 86:1033-1048.

Freeman JA, Nicholson C (1975) Experimental optimization of current source-density technique for anuran cerebellum. J Neurophysiol 38:369-382.

Hallman LE, Schofield BR, Lin CS (1988) Dendritic morphology and axon collaterals of corticotectal, corticopontine, and callosal neurons in layer $\mathrm{V}$ of primary visual cortex of the hooded rat. J Comp Neurol 272:149-160.

Helmchen F, Svoboda K, Denk W, Tank DW (1999) In vivo dendritic calcium dynamics in deep-layer cortical pyramidal neurons. Nat Neurosci 2:989-996

Kampa BM, Johannes J, Stuart GJ (2006) Requirement of dendritic calcium spikes for induction of spike-timing-dependent synaptic plasticity. J Physiol (Lond) 574:283-290.

Kasper EM, Larkman AU, Lubke J, Blakemore C (1994) Pyramidal neurons in layer 5 of the rat visual cortex. I. Correlation among cell morphology, intrinsic electrophysiological properties, and axon targets. J Comp Neurol 339:459-474.

Kim HG, Connors BW (1993) Apical dendrites of the neocortex: correlation between sodium- and calcium-dependent spiking and pyramidal cell morphology. J Neurosci 13:5301-5311.

Korngreen A, Sakmann B (2000) Voltage-gated $\mathrm{K}^{+}$channels in layer 5 neocortical pyramidal neurones from young rats: subtypes and gradients. J Physiol (Lond) 3:621-639.

Larkum ME, Zhu JJ (2002) Signaling of layer 1 and whisker-evoked $\mathrm{Ca}^{2+}$ and $\mathrm{Na}^{+}$action potentials in distal and terminal dendrites of rat neocortical pyramidal neurons in vitro and in vivo. J Neurosci 22:6991-7005.

Larkum ME, Kaiser KM, Sakmann B (1999a) Calcium electrogenesis in distal apical dendrites of layer 5 pyramidal cells at a critical frequency of back-propagating action potentials. Proc Natl Acad Sci USA 96:14600-14604.

Larkum ME, Zhu JJ, Sakmann B (1999b) A new cellular mechanism for coupling inputs arriving at different cortical layers. Nature 398:338-341.

Linden DJ (1999) The return of the spike: postsynaptic action potentials and the induction of LTP and LTD. Neuron 22:661-666.

London M, Häusser M (2005) Dendritic computation. Annu Rev Neurosci 28:503-532.

Lorincz A, Notomi T, Tamas G, Shigemoto R, Nusser Z (2002) Polarized and compartment-dependent distribution of HCN1 in pyramidal cell dendrites. Nat Neurosci 5:1185-1193.

Magee JC, Johnston DA (1997) Synaptically controlled, associative signal for Hebbian plasticity in hippocampal neurons. Science 275:209-213.

Markram H, Lubke J, Frotscher M, Sakmann B (1997) Regulation of synaptic efficacy by coincidence of postsynaptic APs and EPSPs. Science 275:213-215.

Paxinos G, Watson C (1997) The rat brain in stereotaxic coordinates, Ed 3. San Diego: Academic.

Rapp M, Yarom Y, Segev I (1996) Modeling back propagating action potential in weakly excitable dendrites of neocortical pyramidal cells. Proc Natl Acad Sci USA 93:11985-11990.

Rudolph M, Destexhe A (2003) A fast-conducting, stochastic integrative mode for neocortical neurons in vivo. J Neurosci 23:2466-2476.

Sawyer CH, Everett JW, Green JD (1954) The rabbit diencephalon in stereotaxic coordinates. J Comp Neurol 101:801-824.

Spruston N, Schiller J, Stuart G, Sakmann B (1995) Activity-dependent action potential invasion and calcium influx into hippocampal CA1 dendrites. Science 268:297-300.

Stuart GJ, Häusser M (2001) Dendritic coincidence detection of EPSPs and action potentials. Nat Neurosci 4:63-71.

Stuart G, Schiller J, Sakmann B (1997) Action potential initiation and propagation in rat neocortical pyramidal neurons. J Physiol (Lond) 505:617-632.

Swadlow HA (1974) Properties of antidromically activated callosal neurons and neurons responsive to callosal input in rabbit binocular cortex. Exp Neurol 43:424-444. 
Swadlow HA (2003) Fast-spike interneurons and feed-forward inhibition in sensory neocortex. Cereb Cortex 13:25-32.

Swadlow HA, Weyand TG (1981) Efferent systems of the rabbit visual cortex: laminar distribution of the cells of origin, axonal conduction velocities, and identification of axonal branches. J Comp Neurol 203: $799-822$.

Swadlow HA, Beloozerova IN, Sirota MG (1998) Sharp, local synchrony among putative feed-forward inhibitory interneurons of rabbit somatosensory cortex. J Neurophysiol 79:567-582.

Swadlow HA, Gusev AG, Bezdudnaya T (2002) Activation of a cortical column by a thalamocortical impulse. J Neurosci 22:7766-7773.
Tsubokawa H, Ross WN (1996) IPSPs modulate spike backpropagation and associated $\left[\mathrm{Ca}^{2+}\right]_{\mathrm{i}}$ changes in the dendrites of hippocampal CA1 pyramidal neurons. J Neurophysiol 76:2896-2906.

Waters J, Helmchen F (2004) Boosting of action potential backpropagation by neocortical network activity in vivo. J Neurosci 24:11127-11136.

Waters J, Schaefer A, Sakmann B (2005) Backpropagating action potentials in neurones: measurement, mechanisms and potential functions. Prog Biophys Mol Biol 87:145-170.

Williams SR, Stuart GJ (2000) Backpropagation of physiological spike trains in neocortical pyramidal neurons: implications for temporal coding in dendrites. J Neurosci 20:8238-8246. 